\title{
Enabling Opportunistic Energy Trading between Overlapping Energy Harvesting Wireless Sensor Networks
}

\author{
Teng Jiang, Geoff V. Merrett, Nick R. Harris \\ Electronics and Computer Science \\ University of Southampton \\ \{tj2g11,gvm,nrh\}@ecs.soton.ac.uk
}

\begin{abstract}
Energy harvesting offers wireless sensor networks the possibility of indefinite operation. Their energy sources are typically uncontrollable and exhibit significant temporalspatial variation. Traditional approaches to managing their operation have been designed to adapt to these variances, but are inherently limited to only managing resources within the physical boundary of the network. Opportunistic Energy Trading (OET) was proposed to allow co-located WSNs to trade energy resources with each other, permitting energy management across network boundaries. In this paper, we extend this prior work by removing a number of simplifying assumptions. We consider the effect of varying degrees of overlap between neighbouring networks, mechanisms for monitoring and recording trades, and the modifications needed at higher layers of the protocol stack. To demonstrate our approach, we evaluate a case study where, during night, a solar-powered network 'buys' energy from a neighbouring battery-powered network to sustain operation.

\section{Categories and Subject Descriptors}

C.2.1 [Computer-Communication Networks]: Network Architecture and Design

Keywords

Wireless sensor networks, energy harvesting, power management, resource scheduling

\section{Introduction}

Wireless Sensor Networks (WSNs) are gaining considerable application in areas pervasive to our daily lives [1]. In some applications, batteries cannot be replaced or recharged, constraining WSNs with a finite lifetime. In these situations, Energy Harvesting WSNs promise prolonged or indefinite operation through the 'harvesting' of energy from ambient sources such as light and vibration [2]. However, the energy available from these sources typically varies in space

Permission to make digital or hard copies of all or part of this work for personal or classroom use is granted without fee provided that copies are not made or distributed for profit or commercial advantage and that copies bear this notice and the full citation on the first page. Copyrights for components of this work owned by others than ACM must be honored. Abstracting with credit is permitted. To copy otherwise, or republish, to post on servers or to redistribute to lists, requires prior specific permission and/or a fee. Request permissions from Permissions@acm.org.

ENSSys'14, November 6, 2014, Memphis, TN, USA.

Copyright (C) 2014 ACM 978-1-4503-3189-0/14/11 ...\$15.00

http://dx.doi.org/10.1145/2675683.2675688
\end{abstract}

and time [3]; for example some nodes in a solar-powered network will harvest more than others due to orientation and shading (spatial variation), while no nodes harvest energy during the night (temporal variation).

Traditional energy-neutral algorithms exploit temporal variation through dynamic node operation $[4,5]$, for example by throttling activity when energy is reduced or by using energy storage (e.g. a supercapacitor) to buffer energy and provide an 'average' performance at all times. To accommodate spatial variation, some approaches attempt to physically transfer energy around the network $[6,7]$, while the majority consider the logical redistribution of energy (where energy-consuming tasks, such as packet routing, are distributed across the network to balance consumption) [8].

However, the tolerable spatial variation is limited by the network boundary. In our previous work [9], we proposed the concept of Opportunistic Energy Trading (OET), where co-located networks could logically share energy resources with each other. Through this, networks can manage spatial energy variation by distributing tasks not only across their own network, but also across others in their locality. This was demonstrated through the simulation of a solar-powered WSN co-located with a battery-powered WSN. During daytime, the battery-powered network routed packets via the solar-powered network (which harvested more power than it used), and increased its network lifetime. The two networks were configured to be fully-overlapping, that is each node in one network had a one-hop connection to a node in the other network.

In this paper, we extend our previous work to move OET closer to reality through the following contributions: 1) a framework for enabling OET at higher layers of the protocol stack to provide required services, 2) a mechanism and metric to allow trading, i.e. monitoring and recording the cost of provided services, 3 ) evaluating our approach through a simulated case study where a solar-powered network can buy energy from a neighbouring battery-powered network overnight, and 4) systematically evaluating the effect on OET of varying degrees of overlap between co-located networks, from no-overlap to fully-overlapping.

\section{Opportunistic Energy Trading (OET)}

In WSNs, target lifetimes can typically be defined as either indefinite (met through energy-neutrality), or definite (meeting a target lifetime). As previously explained, tradi- 
tional power-management strategies address these by temporally adjusting a node's operation(accommodating temporal energy variation), and/or balancing energy consumption across nodes (accommodating spatial energy variation). These can be ineffective as:

- Nodes have finite energy storage. Excess energy is typically wasted, often through an unnecessary increase in duty cycle, transmitting packets that do not provide any additional information. As systems miniaturise, energy storage significantly reduces, further exacerbating this;

- Spatial energy management algorithms can only balance energy across the nodes in WSN, and are hence limited by the harvesting modality and its spatial variability within its own network bounds.

OET permits co-located WSNs to logically trade energy resources with each other, potentially enabling greater flexibility than intra-network techniques. Two previously unaddressed challenges of OET are reported on in this paper. First, co-located networks need to opportunistically interconnect with one another, including both the process of discovery (that is, they are not pre-programmed with knowledge of neighbouring networks) and can communicate without a dedicated gateway. This has previously been considered at the lower-layers of the protocol stack [10]. However, support in the upper-layers of the protocol stack (e.g. routing and application) has not been considered. Second, for energy trading to be adopted, the cost of the services that they provide and use needs to be quantified, monitored and recorded. In previous work [9], only zero-cost trades (or energy 'sharing') were considered.

The remainder of this section explains the proposed framework for OET which addresses the above challenges.

\subsection{A framework for OET support}

'Interconnection' of two networks requires two things. First, packets need to be communicated from one network to another; second, the information contained within a packet should be understood by both networks (clearly, this raises privacy and security issues; these are outside the scope of this work and remain areas of future investigation). The first of these requirements was addressed in OI-MAC, which showed how to achieve this in the lower layers of the protocol stack. Full details of OI-MAC, which we build upon in this paper, are omitted here for conciseness; readers are instead referred to [10]. The second requirement, interpretting the meaning of a packet, is addressed here through modifications to the upper-layer protocols of the stack (illustrated in Fig. 1) This requires standardised modules in both the routing and application layers (here, standardised refers to the data format and information definition being the same, regardless of the protocol that they are attached to).

Traditionally, application layers do not need to consider cross-boundary information and resource sharing. As neighbouring networks may use different protocols, they will likely use different formats to define and represent information and resources. Essential for OET, different networks need to know some basic parameters about each other (e.g. the services provided and available resources) before they can cooperate. Therefore, two standardised modules are

\begin{tabular}{|c|c|}
\hline $\begin{array}{c}\text { Service Management Module, } \\
\text { Resource Management Module }\end{array}$ & Application layer \\
\hline Addressing Translation Module & Network Layer \\
\hline \hline OIMAC & \\
\hline \hline IEEE 802.15.4 PHY \\
\hline IEEE 802.15.4 Radio Chip \\
\hline
\end{tabular}

Figure 1. Framework for OET support in a protocol stack using IEEE 802.15.4 and OI-MAC. Shaded blocks indicate standardized modules.

appended to the application layer: the Service Management Module (SMM) and Resource Management Module (RMM). The SMM records and advertises services that a network can provide. The RMM describes available resources (e.g. energy, network coverage). Based on information in these two modules, neighbouring networks can decide when to start and stop cooperation.

OET is a logical trade of energy, that is energy is traded by offloading energy-consuming tasks to other networks. While the RMM and SMM allow networks to understand the services offered by neighbouring networks, a deeper understanding of the meaning of transferred packets is required for tasks to actually be offloaded. While a range of offload able energy consuming tasks exist (e.g. computation, connected services, sensing etc), here we consider only the offloading of packet routing. As neighbouring networks may use different protocol stacks, and hence routing algorithms, a mechanism is needed for them to understand the meaning of routing requests. Therefore, for every cross-boundary transmission, the Addressing Translation Module (ATM) encapsulates packets with a standardized Cross-Boundary Addressing (CBA) header. When a boundary node receives a packet from a neighbouring network, it translates the CBA header into its local routing header. When transmitting a packet back across the boundary, it regenerates the CBA header.

A typical CBA routing header is shown in Fig. 2. The Frame Control field includes information on the frame type, time stamp, security etc. The Passport ID field contains information set during the handshaking process and related to cooperation, for example routes selected, the duration of cooperation, and other relevant parameters etc. This information tells nodes how to serve different packets, for example which route to use. During the translation process, the Passport ID remains unchanged and is included in the local routing header.

One final cross-layer module, named the Cooperation Management Layer (CML), is added to the stack to support OET. The CML has two functions. First, it is responsible for collecting information from each layer (e.g. cost related metrics); second, it can configure the parameters of the MAC and routing layers in order to provide required services.

\subsection{Monitoring and recording cost}

Central to the concept of OET is that of trading. In order to transact a trade, each network must be able to record the 


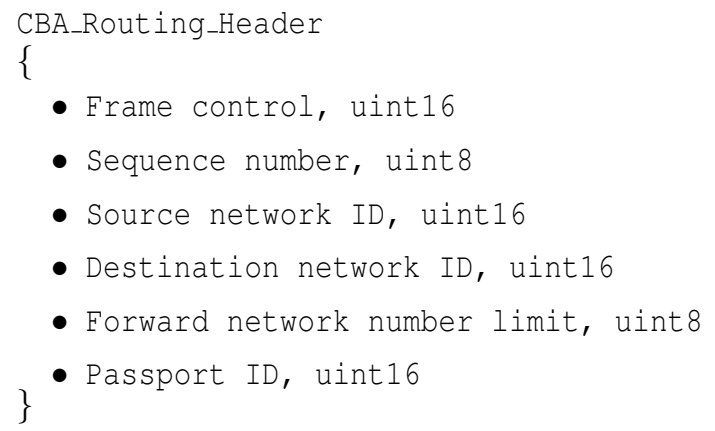

Figure 2. Standardized Cross-Boundary Addressing (CBA) header for cross-boundary transmissions

resources that it invested in the cooperation, such that this can be translated into a cost. Trades may be undertaken on the basis of fairness (i.e. networks are symbiotic, and use an equal measure of each others resources), or for a cost (i.e. a monetary transaction conducted by the stakeholders). A sensible metric to quantify the cost of resources is the amount of energy consumed while routing the neighbouring network's packets. Here, we assume a proxy of this, namely the total number of hops taken to route the packet. This is calculated by $\sum_{i=1}^{N} H_{i}$, where $H_{i}$ is the total number of hops needed by packet $i$ before reaching its destination, and $N$ is the total number of packets from the neighbouring network that have been routed. Each node keeps a record of how many packets it has transmitted for the neighbouring network. This information is then transmitted back to the management node (e.g. the sink) where it can be translated into an energy cost.

\section{Results: an illustrative case study}

To illustrate OET, we evaluate its operation through a simulated case study. This case study is considered to evaluate the feasibility of the approach illustrated in the previous section, but also to demonstrate the potential benefits and effects of OET on both networks.

\subsection{Case study scenario}

Network A and B are two 25-node networks, where nodes are organised in a grid structure (this assumption is made to allow easier interpretation of results, and is not a requirement of OET). In our previous work, we considered that the networks were fully-overlapped, that is each node had a corresponding one-hop link to a node in the other network. To remove this simplifying assumption, here we evaluate the effect of varying degrees of overlap. To do this, an overlap area ranging from $0 \%$ (the two networks are independent and cannot support OET) to $100 \%$ (fully-overlapped) are simulated, as shown in Fig. 3). This overlap percentage is calculated as the ratio of the number of nodes in the overlap area to the total number of nodes in the network. For simplicity, we assume that the sink of Network A is always in the overlap area (where one exists), and hence one hop from Network B.

The nodes in Network A are powered by an on-board solar-cell (we model a $3.3 \mathrm{~cm} \times 6.35 \mathrm{~cm}$ photovoltaic being used to recharge a $15 \mathrm{~F} / 2.7 \mathrm{~V}$ supercapacitor). The available solar energy is modelled using the radiation power data provided by Humboldt State University $\left(40.99^{\circ} \mathrm{N}, 124.08^{\circ} \mathrm{W}\right.$,

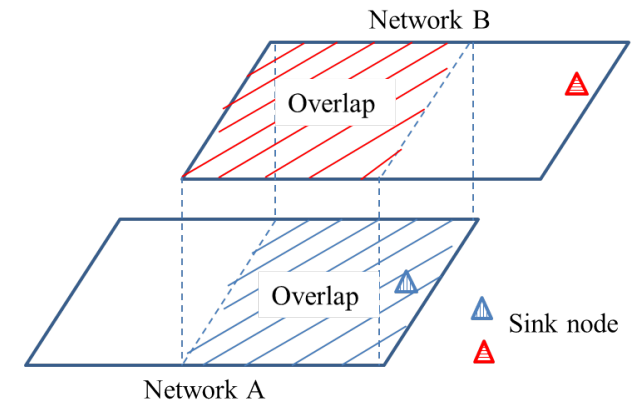

Figure 3. The relative positions of overlapping networks.

elevation 36m) between 07-11 January. Each photovoltaic is assumed to be $10 \%$ efficient, which is typical of off-theshelf cells ${ }^{1}$. The nodes in Network B are powered by a $2400 \mathrm{mAh} / 3 \mathrm{~V}$ non-rechargeable battery. Nodes in both networks sample data every $120 \mathrm{~s}$.

\subsection{Protocol modification and implementation}

The implementation of the protocol stack was undertaken following the design specified in section 2.1 and shown in Fig. 1. For ease of comparison, the PHY and MAC layers are configured the same as that reported in [10]. After discovering each other, the sinks from both networks exchange application-level messages and create a Passport ID to represent the current strategy for cooperation. After this, both sinks broadcast information to every node in their network.

Both networks use the collection tree routing protocol. For simplicity, and to enable intuitive evaluation, routing tables are not updated. Therefore, when a node's route to the sink fails as a result of an intermediate hop running out of energy, it will be unable to communicate with the sink any more (clearly this routing algorithm is not energy-aware, but is used here for illustration). However, unlike a traditional intra-network routing algorithm, each node maintains two routing tables: one records the shortest intra-network route to the sink (in-route), while the other records the closest route to the neighbouring network (ex-route). When a node decides to send a packet via the neighbouring network, it attaches a Passport ID to the routing header and uses the exrouting table to select the next hop. If both routes are not available, the packet will be dropped ${ }^{2}$.

As described in section 2.1, the CML not only acts as a database to store cost related data, but also provides a 'bridge' for the lower layers to provide information to the upper layers. In our implementation, if the MAC layer fails to communicate a packet to a node twice, it assumes that the next hop has died and notifies the CML. The CML adjusts the behaviour of routing layer based on this feedback.

\subsection{Strategy for OET-based cooperation}

If a node in Network $A$ is unable to use its established route for sending a packet to the sink, it will instead transmit it towards the boundary. If the next node can route it using its own in-route, it does. Otherwise, it continues to be routed

\footnotetext{
${ }^{1}$ Our modelled solar irradiance does not vary spatially.

${ }^{2}$ If routing tables are updated dynamically at run-time, change should be made to both.
} 


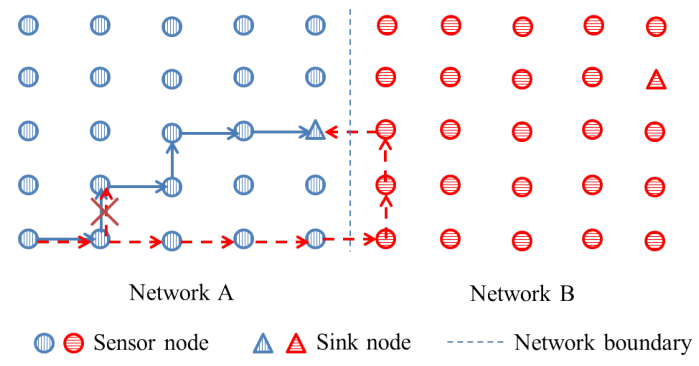

Figure 4. When available, an in-route path is taken (solid). Otherwise, the ex-route path is taken (dashed).

towards the boundary (shown in Fig. 4). On Network B's receipt of the packet at the boundary, it routes the packet towards the boundary node closest to the sink in Network A 3 .

Network A is configured with an infinite budget, that is it will buy Network B's energy resources whenever they are needed (likewise, Network B will happily sell its energy resources at any point). Cost data on the number of transmissions sold are piggybacked to Network B's sink node along with its own routine data packets.

\section{Simulation results}

The case study scenario was implemented using OM$\mathrm{NeT}++[11]$. Results from each simulation are analysed over 20 simulation runs. Simulation parameters related to the PHY/MAC and wireless channel are the same as that presented in [10]. To evaluate the impact of network overlap on the performance of OET, overlap percentages of $0 \%, 20 \%$, $40 \%, 60 \%, 80 \%$ and $100 \%$ are considered.

\subsection{Packet delivery}

Fig. 5 shows the effect of network overlap on the number of packets successfully transmitted by Network A (solarpowered). It can be seen that, without any overlap and hence without OET, the majority of packets are lost overnight (e.g. the flat line during $0.9<T<1.2$ ) as routes between sensor nodes and the sink deplete their energy stores. As the overlap percentage increases, the number of packets successfully received increases, as each node has alternative routes via the neighbouring network which it can utilise.

To further illustrate the effect of OET on packet reception, Fig. 6 shows how many packets each node in the network successfully delivers. The current OET strategy does not bring improvement to the packet delivery of nodes located along the middle row, as their closest boundary node lies behind the sink node; hence they only have a single route. In general however, nodes benefit significantly from OET as they reduce their dependence on in-routes. Considering node $(1,1)^{4}$ as an example, the number of packets delivered increases by more than $100 \%$ when the overlap percentage increases from $0 \%$ to $100 \%$.

Fig. 7 shows the effect of OET on packets in Network B (battery-powered), i.e. the potential detrimental effect of

\footnotetext{
${ }^{3}$ In the current implementation, the CBA header does not contain the address of a specific node in the neighbouring network; instead the destination of neighbour's packets are always assumed to be its sink.

${ }^{4}$ Nodes are indexed in the format (row, column).
}

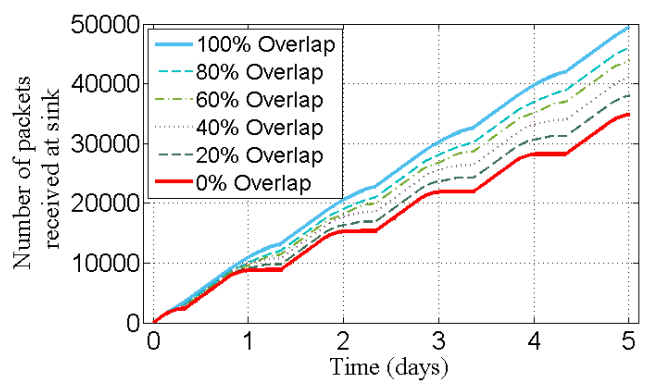

Figure 5. Number of packets successfully delivered in Network A (solar-powered) with varying overlap.

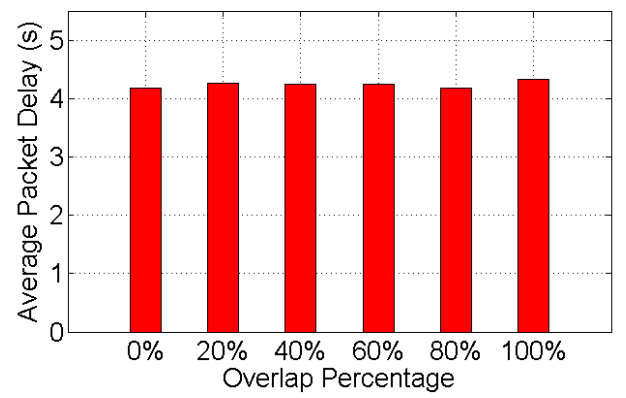

Figure 7. Average packet delay in Network B (batterypowered) with varying overlap percentages.

routing its neighbours packets on routing its own packets. The effect is insignificant $(<2 \%)$, and any variation is likely due to the non-determinism of individual simulations (even after averaging across 20 runs). This is because OI-MAC allows for a sequence of data transmissions within a single duty cycle (refer to [10] for a detailed evaluation of OIMAC's performance on packet delivery).

\subsection{Residual energy}

Fig. 8 shows how the energy in Network A's (solarpowered) nodes changes through time. The max, min and mean are shown to illustrate how the energy varies across nodes. As intermediate overlap percentages translate into intermediate residual energy results, the two extremes of $0 \%$ and $100 \%$ overlap are presented for brevity. As shown, some nodes deplete their energy stores and die during the night (e.g. $0.9<T<1.2$ ). Without OET, energy remains that cannot be utilised during the night (up to $30 \%$ ), as nodes furthest from the sink become isolated as routing nodes die. After adopting OET, alternative route selection evens out energy consumption, improving energy utility.

To further illustrate this, the residual energy in Network A (solar-powered) during the night is shown in Fig. 9. When there is no overlap, and hence no OET, node $(4,4)$ and $(2,4)$ have around $14 \mathrm{~J}$ left at the end of the night as routing nodes $(3,4),(4,5)$ and $(2,5)$ deplete. With $20 \%$ overlap, boundary nodes $(5,1)(5,2),(5,4),(5,5)$ are all depleted at the end of the night as more packets have been transmitted towards them along ex-route paths after the in-route paths become unavailable. The effect of this is increased as the overlap area increases; that is nodes under the overlap area have a higher 


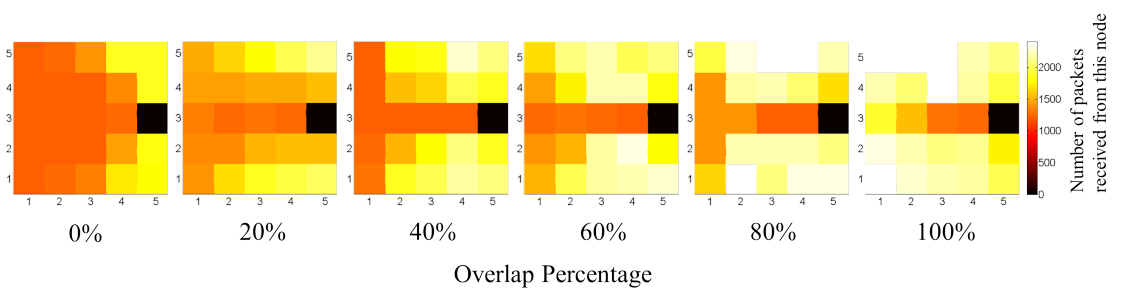

Figure 6. The number of packets successfully delivered by each node in Network A (solar-powered) with varying overlap percentages. The darkest square indicates the location of the sink, which does not transmit any packets.

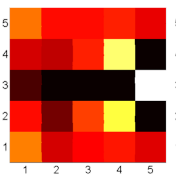

$0 \%$

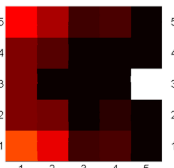

$20 \%$

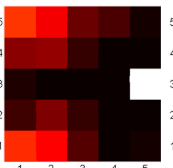

$40 \%$

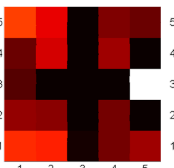

$60 \%$

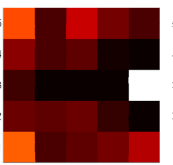

$80 \%$

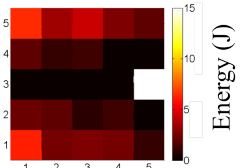

$100 \%$

Overlap Percentage

Figure 9. Residual energy in Network A (solar-powered) at the end of a night. The lightest square indicates the position of the sink, which is assumed to be mains powered.

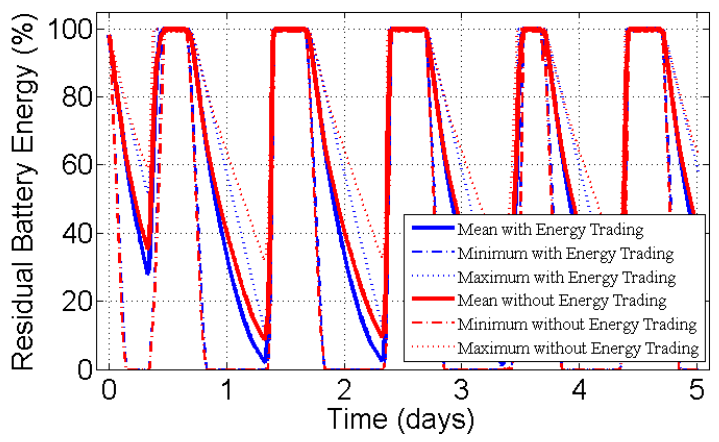

Figure 8. Residual energy in Network A (solar-powered) with and without OET.

energy utility.

For Network B (battery-powered), the impact of OET can be observed during the night when its nodes route packets for Network A (see Fig. 10). After 5 days of simulated operation, OET has decreased the minimum residual battery energy by $2.5 \%$ (extrapolating this, the network lifetime will reduce by around $25 \%$ ). While this has a significant effect on the energy resources of Network B, this energy has been logically transferred to Network A to help it sustain packet transmission during the night. As such, this energy has been traded, or sold, to Network A for which a financial charge should be instigated to provide reimbursement.

This impact of OET on the energy consumption of Network B (battery-powered) can also be seen from Fig. 11, which plots the additional energy consumed by each node to support OET. When overlap is present, some nodes in Network B route packets towards the neighbouring sink, and therefore consume more energy than before. With an overlap of 20\%, the additional energy consumed is not significant $(<1 \%)$, as the number of packets is not considerable. When

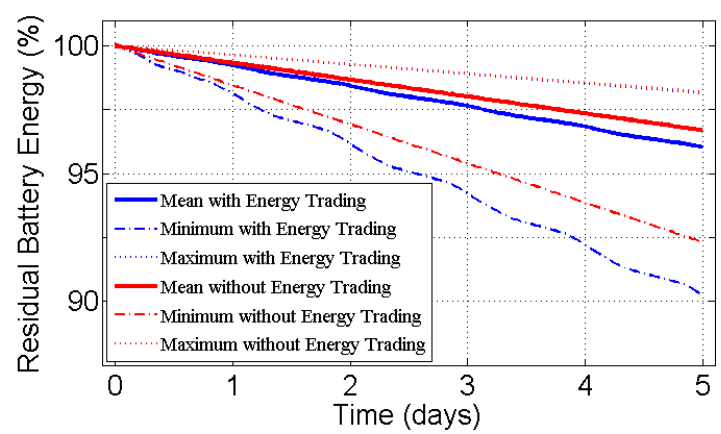

Figure 10. Residual energy in Network B (batterypowered) with and without OET.

the overlap increases, the nodes closest to the neighbouring sink node consume more energy (around 4.5\%). As the additional packet flows affect channel access (e.g. probability of collisions), small variations of $\pm 1 \%$ are to be expected, explaining the 'ripple' on nodes which should not otherwise be affected.

\subsection{Measuring cost}

Fig. 12 plots the cost (number of hops made) recorded by Network B (battery-powered). It can be seen that the gradient, and hence cost, increases with overlap. This is because, when the networks are more overlapped, not only are more packets routed, but also the average number of hops per packet increases (as the distance to the sink increases). From our above observations (Fig. 11), this cost metric appears to be a good approximation of the 'traded' energy.

\subsection{Network topology}

In the above analysis, we have only considered a $5 \times 5$ (square) grid topology. This is a special case, and 'real' networks will likely have non-square topologies, affecting the number of boundary nodes. To evaluate the effect of this, we 


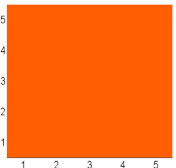

$0 \%$

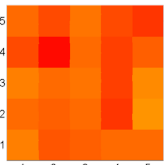

$20 \%$

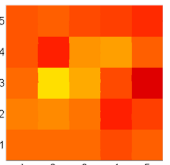

$40 \%$

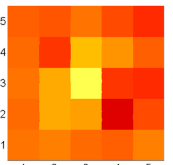

$60 \%$

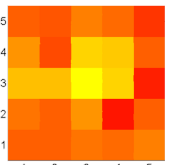

$80 \%$

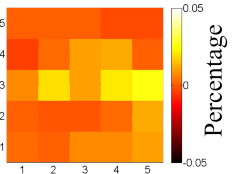

$100 \%$

Overlap Percentage

Figure 11. Change in residual energy in Network B (battery-powered) at the end of the simulation.

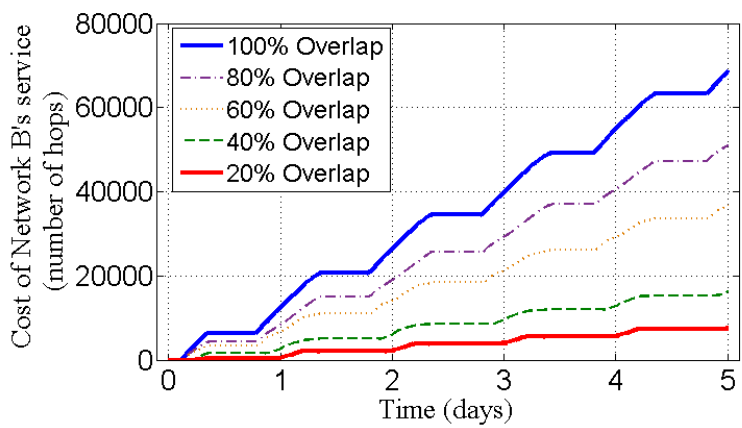

Figure 12. Cost of OET recorded by Network B.

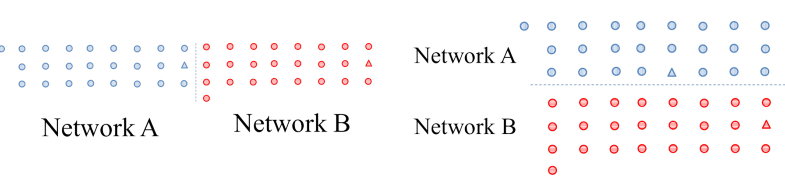

(a)

(b)

Figure 13. Considered topologies, (a) $8 \times 3$, (b) $3 \times 8$.

simulated $3 \times 8$ and $8 \times 3$ topologies (illustrated by Fig. 13). Fig. 14 shows the number of packets successfully delivered in Network A for each topology, when the boundary is only one-node thick (i.e. $20 \%$ in the case of the $5 \times 5$ network). These results agree with our previous conclusions: the more 'overlapped' the networks are, the higher the proportion of boundary nodes, and hence the more effective OET is.

\section{Conclusions}

This paper has illustrated how OET allows energy to be traded between overlapping WSNs. This has extended our previous work through a framework for enabling OET at higher layers of the protocol stack, including support for services to monitor and record the cost of trades. A case study consisting of a solar-powered network buying energy from a battery-powered network was used to illustrate the approach. Furthermore, the effect of varying degrees of overlap was evaluated. As expected, OET enabled the solar-powered network to offload energy-consuming tasks, and the benefit of this increased as the overlap increased. OET has little impact on the battery-powered network, except for energy consumption (which is recorded so that the stakeholder can be financially reimbursed).

In our future work, we will utilise energy-aware routing algorithms and consider bi-directional OET, whereby networks can use accumulate cost to buy back services. We

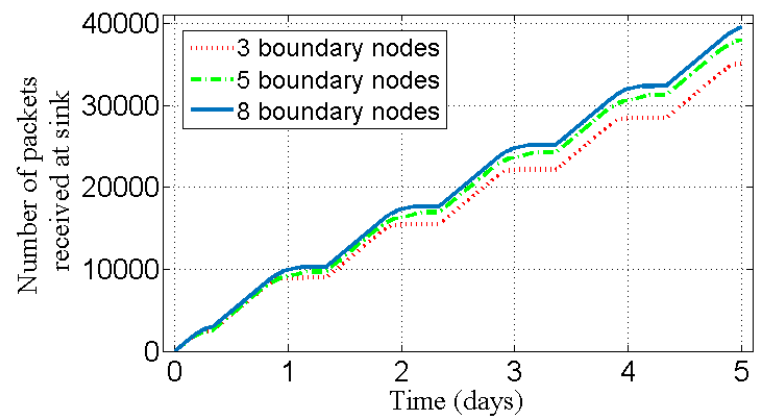

Figure 14. Number of packets received in Network A when adopting different topologies.

are also working towards a practical implementation of OET, with real scenarios and topologies.

\section{References}

[1] Jennifer Yick et al. Wireless sensor network survey. Computer networks, 52(12):2292-2330, 2008.

[2] S. Beeby and N. White. Energy Harvesting for Autonomous Systems. Artech House series smart materials, structures, and systems. Artech House, Incorporated, 2010.

[3] Ren-Shiou Liu et al. Joint energy management and resource allocation in rechargeable sensor networks. In INFOCOM, pages 1-9, 2010.

[4] Christian Renner et al. Adaptive energy-harvest profiling to enhance depletion-safe operation and efficient task scheduling. Sustainable Computing: Informatics and Systems, 2(1):43-56, March 2012.

[5] Zhoujia Mao et al. Near optimal power and rate control of multi-hop sensor networks with energy replenishment: Basic limitations with finite energy and data storage. IEEE Transactions on Automatic Control, 57(4):815-829, 2012.

[6] Sheng Zhang et al. Collaborative mobile charging for sensor networks. In Conf on Mobile Adhoc and Sensor Systems, pages 84-92, 2012.

[7] Ting Zhu et al. Eshare: a capacitor-driven energy storage and sharing network for long-term operation. In Proceedings of the 8th ACM Conference on Embedded Networked Sensor Systems, pages 239-252. ACM, 2010.

[8] Zhi Ang Eu et al. Opportunistic routing in wireless sensor networks powered by ambient energy harvesting. Computer Networks, 54(17):2943-2966, 2010.

[9] Teng Jiang et al. Opportunistic energy trading between co-located energy-harvesting wireless sensor networks. Proceedings of the 1st Int'l Workshop on Energy Neutral Sensing Systems, page 11, 2013.

[10] Teng Jiang et al. Opportunistic direct interconnection between colocated wireless sensor networks. 22nd Int'l Conference on Computer Communications and Networks, pages 1-5, 2013.

[11] Andreas Köpke et al. Simulating wireless and mobile networks in omnet++ the mixim vision. In Int'l Conf on Simulation tools and techniques for communications, networks and systems \& workshops, page 71. ICST, 2008. 